\title{
Hayabusa2: Sample Acquisition at a Near-Earth C-type Asteroid Ryugu and Analysis Plan of Returned Samples
}

\author{
Shogo Tachibana ${ }^{1}$ \\ 1. UTokyo Organization for Planetary and Space Science (UTOPS), University of Tokyo, 7-3-1 Hongo, \\ Tokyo 113-0033, Japan. \\ * Corresponding author: tachi@eps.s.u-tokyo.ac.jp
}

Hayabusa2, JAXA's asteroidal explorer, arrived at a near-Earth C-type asteroid (162173) Ryugu on June 27, 2018. Ryugu is expected to record a long evolutionary history of the Solar System from the beginning to the present [1]. Since its arrival, Hayabusa2 has been investigating the asteroid [2-4] with a telescopic optical camera with 7 band filters (ONC-T) [5], a near-infrared spectrometer (NIRS3) [6], a thermal infrared imager (TIR) [7], and a laser altimeter (LIDAR) [8], two rovers (MINERVA-II1; HIBOU and OWL), and a MASCOT lander [9].

It has been found that Ryugu has a top shape with an equatorial ridge (mean radius of $448 \pm 2 \mathrm{~m}$ ), having a retrograde rotation with a period of 7.6326 hours and an obliquity of $172^{\circ}$ [2] (Fig. 1). Its bulk density is estimated to be $1.19 \pm 0.03 \mathrm{~g} / \mathrm{cm}^{3}$, suggesting that the asteroid has a large macro-porosity of $\sim 50-60 \%$ [2]. One of the striking surface geological features is the presence of many large $(>20 \mathrm{~m})$ boulders with a number density twice as large as that of Itokawa, and there is no smooth terrain as seen in Itokawa [3]. These observations (low bulk density and boulder-rich surface) lead to the conclusion that Ryugu is a rubble-pile body [2]. It has been also found that the surface has a very low geometric albedo, darker than most of meteorite samples [3], and that the surface has uniformity in visible and near infrared spectra with a weak $2.72-\mu \mathrm{m}$ absorption feature $[3,4]$. The $2.72 \mu \mathrm{m}$ absorption feature suggests the ubiquitous presence of hydrous phases [4].

The Hayabusa2 sample acquisition system (Hayabusa2 sampler) will shoot a 5-g tantalum projectile inside a 1-m long sampler horn at an impact velocity $300 \mathrm{~m} \mathrm{~s}^{-1}$ when the spacecraft lands. The ejecta going up through the sampler horn will be stored in a sample catcher located at the top end of the sampler horn. Three projectiles are equipped for sample acquisition at three different surface locations. The bottom end of the sampler horn is turned up to lift up surface pebbles $(<\sim 5 \mathrm{~mm})$ during landing. The lifted pebbles will be stored in the sample catcher by deceleration of the spacecraft.

The sample catcher (total volume $\sim 45 \mathrm{~cm}^{-3}$ ) has three chambers to store samples obtained at three locations separately [10]. The sample catcher will be sealed in the sample container inside the Earth reentry capsule with an aluminum metal seal to avoid the terrestrial air contamination [11]. The container sealing system allows only a leak of $1 \mathrm{~Pa}$ air for 100 hours in the air. Soon after the capsule recovery, volatile components inside the sample container, which could be released from the samples, will be extracted before the container opening operation using a newly developed gas extraction line.

The first landing for sample acquisition was originally planned to be in October 2018, but due to the presence of many boulders it was postponed to February 2019. On February 22, Hayabusa2 successfully landed Ryugu for sample acquisition (Fig. 1). It was also confirmed that the projectile was shot as expected. Detailed analysis of images taken during the landing operation will be done to estimate the amount of ejecta that could be a measure of the samples collected and to discuss properties of surface 
materials.

The reentry capsule will be delivered to the Earth in late 2020. The sample container inside the capsule will be opened in vacuum after extraction of volatile components released in the sampler container. A small fraction of the samples will be picked up in vacuum to store for future generation. The rest of the samples in the sample catcher will be handled in dry nitrogen atmosphere without exposure to the air. All these curation work will be done at Institute of Space and Astronautical Science, JAXA. A fraction of the samples will then be distributed to the Hayabusa2 team for the one-year initial sample analysis. The initial analysis will be science-oriented to characterize the Ryugu samples and to understand the history of Ryugu and the Solar System. The initial analysis team consists of six international sub-teams with different disciplines and targets: 1) chemistry, 2) petrology and mineralogy of coarse grains, 3) petrology and mineralogy of fine grains, 4) volatiles, 5) macromolecular organics, and 6) organic molecules. Microanalysis is a key technology that provides essential information from limited amounts of returned samples.

References:

[1] Tachibana S. et al. Geochem. J. 48, 571-587.

[2] Watanabe S. et al., Science doi 10.1126/science.aav8032.

[3] Sugita S. et al., Science doi 10.1126/science.aaw0422.

[4] Kitazato K. et al. Science doi 10.1126/science.aav7432.

[5] Mizuno T. et al. (2017) Space Sci. Rev. 208, 33-47.

[6] Kameda S. et al. (2017) Space Sci. Rev. 208, 17-31.

[7] Iwata T. et al. (2017) Space Sci. Rev. 208, 317-337.

[8] Okada T. et al. (2017) Space Sci. Rev. 208, 125-142.

[9] Ho T.-M. et al. (2017) Space Sci. Rev. 208, 339-374.

[10] Sawada H. et al. (2017) Geochem. J. 48, 81-106.

[11] Okazaki R. et al. (2017) Geochem. J. 48, 107-124.

[12] The author acknowledges the Hayabusa2 Sampler Team and the Sample Analysis Team for development of the Hayabusa2 sampler and planning of the sample analysis.

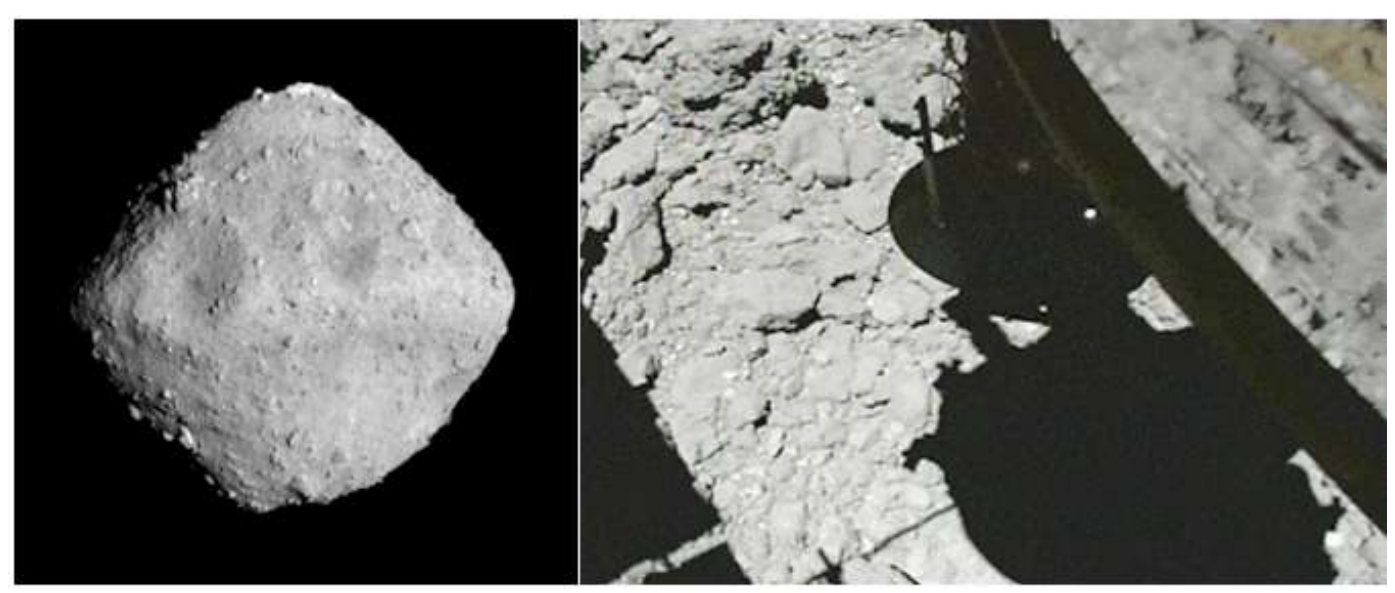

Figure 1. (162173) Ryugu (left) and the surface of Ryugu taken during the first landing operation (right). (c) JAXA, Univ. Tokyo, Kochi Univ., Rikkyo Univ., Nagoya Univ., Chiba Inst. Tech., Meiji Univ., Univ. Aizu, AIST. 